\title{
Imunização ao desamparo aprendido após reforçamento positivo em ratos ${ }^{1}$
}

\author{
Maria Helena Leite Hunziker \\ Raquel Scarpa Gebara Garcia de Lima \\ Universidade de São Paulo
}

\begin{abstract}
RESUMO
Este estudo investigou se a exposição a diferentes contingências de reforçamento positivo, antes do tratamento com choques incontroláveis, pode impedir o desenvolvimento do desamparo aprendido em ratos (efeito de imunização). Foram manipulados cinco grupos de ratos Wistar $(n=8)$. Sujeitos de três grupos foram submetidos a sessões de reforçamento positivo (CRF, FR6 ou múltiplo-concorrente FR 6/Extinção), seguidas de uma sessão de choques incontroláveis e outra de teste de fuga. Sujeitos de outro grupo foram expostos apenas a sessões de choques incontroláveis e teste de fuga, sendo que os animais do grupo restante foram expostos apenas à sessão de teste de fuga. Os resultados no teste mostraram que apenas os animais submetidos previamente aos choques incontroláveis apresentaram dificuldade na aprendizagem de fuga (desamparo aprendido). Contudo, aqueles expostos inicialmente ao reforçamento positivo tiveram reduzido o efeito dos choques incontroláveis, independente da contingência utilizada. Esses resultados sugerem que a exposição repetida ao reforçamento positivo pode produzir o efeito de imunização.
\end{abstract}

Palavras-chave: desamparo aprendido; efeito de imunização; controle aversivo

\begin{abstract}
Immunization to learned helplessness after Positive Reinforcement in Rats

This study investigated whether the exposure to different positive reinforcement contingencies, prior to the uncontrollable shock treatment, can reduce the learned helplessness effect in rats (immunization). Five groups of Wistar rats $(n=8)$ were used. Animals from three groups were exposed to different positive reinforcement schedules (CRF, FR 6 or multiple-concurrent), followed by a session of uncontrollable shock and an escape test session; animals from the fourth group were submitted only to uncontrollable shocks and escape test session; subjects in the last group were submitted only to the escape test session. Only the subjects exposed to uncontrollable shock treatment had difficulty to learn the escape response during the test session (learned helplessness effect). However, rats initially exposed to different positive reinforcement contingencies showed reduced escape latencies. These results suggest that repeated exposure to positive reinforcement, before uncontrollable shock treatment, can produce the immunization effect.
\end{abstract}

Keywords: learned helplessness; immunization; aversive control

Uma história comportamental que envolve estímulos aversivos incontroláveis produz, posteriormente, dificuldade de aprendizagem, sendo esse efeito denominado desamparo aprendido (Maier \& Seligman, 1976; Peterson, Maier \& Seligman, 1993). Contudo, o desamparo não é obtido se anteriormente à condição de incontrolabilidade o sujeito for submetido a estímulos aversivos que podem ser controlados pelo seu comportamento. Tal efeito preventivo ao desamparo vem sendo chamado de imunização (Maier \& Seligman, 1976).
A interpretação mais difundida sobre os efeitos de desamparo e de imunização propõe que é a primeira condição de estímulo que determina o comportamento posterior (Maier \& Seligman, 1976). Assim, se a primeira exposição for a estímulos incontroláveis, o sujeito vai aprender que a alteração nos estímulos independe do seu comportamento, o que impediria posteriormente a aprendizagem operante, produzindo o desamparo. Por outro lado, se a primeira experiência for com estímulos controláveis, o sujeito aprende que os estímulos podem ser alterados pelo seu comporta- 
mento, de forma que a exposição posterior a estímulos incontroláveis não interfere na aprendizagem operante, caracterizando o efeito de imunização.

Os estudos sobre imunização não variaram o tipo de estímulo manipulado, sendo utilizado basicamente o choque elétrico, tanto controlável como incontrolável, nas diferentes fases da pesquisa (Alloy \& Bersh, 1979; Anisman, Irwin, Beauchamp \& Zacharko, 1983; Brown, Howe \& Jones, 1990; Kirk \& Blampied, 1986; Ramirez, Maldonado \& Matos, 1992; Seligman, Rosellini \& Kozak, 1975; Yano \& Hunziker, 2000). Essa invariância nas pesquisas permite que se tenha ao menos duas interpretações sobre o que imuniza o sujeito contra o desamparo: (1) a experiência de controle sobre qualquer estímulo antes da exposição à condição de incontrolabilidade; (2) a experiência de controle sobre estímulos semelhantes aos que posteriormente serão experimentados como incontroláveis. Cada uma dessas interpretações tem implicações distintas: a primeira sugere que qualquer aprendizagem operante pode produzir o efeito de imunização; a segunda limita esse efeito apenas às condições onde os estímulos são iguais entre si nas diferentes fases do estudo. Dada a enorme diferença das implicações teóricas de ambas, torna-se muito relevante que elas sejam confrontadas experimentalmente.

Alguns poucos estudos variaram os estímulos apresentados aos sujeitos, principalmente no que diz respeito à sua função aversiva, sendo conflitantes os resultados descritos. Por exemplo, Mestre e Hunziker (1996) observaram que sujeitos submetidos a uma sessão de reforçamento positivo da resposta de pressão à barra (modelagem, seguida de alguns reforços em esquema de reforçamento contínuo - CRF), e depois ao tratamento com choques incontroláveis, apresentaram, posteriormente, dificuldade de aprender fuga frente a novos choques (desamparo). Efeito contrário foi obtido por Hunziker, Manfré e Yamada (2006), que utilizando reforçamento positivo/choques incontroláveis/fuga, obtiveram efeito de imunização. Contudo, diferentemente do estudo anterior, Hunziker e cols. utilizaram, na primeira fase, 10 sessões de reforçamento positivo contingente à variação ou à repetição de sequiências de quatro respostas de pressão a duas barras.

Duas diferenças nos procedimentos desses estudos podem ser apontadas como possivelmente críticas para se compreender os resultados opostos: a quantidade de sessões de reforçamento positivo e o tipo de contingência utilizada. No geral, as pesquisas sobre imunização oferecem ao sujeito, na fase inicial, uma única sessão de reforçamento negativo onde se exige a emissão de apenas uma resposta para desligar o choque. Nesse sentido, o procedimento de Mestre e Hunziker (1996) é, dentre os dois acima citados, o mais comparável à maioria dos estudos sobre imunização. Contudo, pode-se argumentar que água, utilizada como reforçador positivo, não tem a mesma magnitude reforçadora da remoção do choque elétrico. Se assim for, é possível supor que um maior número de sessões de reforçamento positivo seja mais comparável a uma única sessão de reforçamento negativo, o que dá ao estudo de Hunziker e cols. maior semelhança com as pesquisas da área. Essas especulações apontam para a necessidade de mais estudos sobre o efeito de imunização em contextos de estímulos não simétricos, tais como aversivos e não aversivos. Investigar essa assimetria permitirá que se identifique a amplitude de generalização que pode ser atribuída ao efeito de imunização.

O presente experimento foi proposto com o objetivo colaborar na resposta a essa questão, investigando se uma primeira experiência com reforçamento positivo pode evitar que a exposição a choques incontroláveis produza o déficit de aprendizagem de fuga. Em função do anteriormente exposto, o reforçamento positivo será apresentado em sessões sucessivas, utilizando-se diferentes de contingências que variam quanto à complexidade do esquema: simples (contínuo ou intermitente) ou complexo (múltiplo/concorrente).

\section{MÉTODO}

\section{Sujeitos}

Foram utilizados 40 ratos Wistar, machos, ingênuos experimentalmente, de aproximadamente três meses de idade, oriundos do Biotério Central da Unicamp. Os sujeitos ficaram alojados no biotério do Departamento de Psicologia Experimental da USP, por um período de dois meses antes do início do experimento, mantidos em gaiolas individuais, com controle de ciclo luz-escuro (7-19h), tendo água e ração balanceada constantemente disponíveis. Apenas os sujeitos que passaram por reforçamento positivo foram mantidos em regime de privação de água $36 \mathrm{~h}$ antes do início da primeira sessão; nas sessões sucessivas, esses animais tinham livre acesso à água por $5 \mathrm{~min} / \mathrm{dia}$ depois de transcorrido um intervalo de 10 minutos do término da sessão. Todos os sujeitos foram pesados diariamente durante o período de coleta de dados como forma de acompanhar seu estado de saúde. 


\section{Equipamento}

Uma das caixas experimentais era de fabricação FUNBEC, medindo 24,0 × 20,0 × 19,0 cm, tendo no centro da parede direita uma barra cilíndrica de $4,0 \mathrm{~cm}$ de comprimento e $1,0 \mathrm{~cm}$ de diâmetro, distando $8,0 \mathrm{~cm}$ do assoalho. Uma pressão de, no mínimo, 45 gf (grama-força) produzia o registro da resposta. Um bebedouro, situado abaixo da barra e o nível do piso, tinha capacidade de liberar $0,05 \mathrm{cc}$ de água. Duas outras caixas, medindo $21,5 \times 21,5 \times 21,0 \mathrm{~cm}$, continham, cada uma, duas barras de $4,0 \mathrm{~cm}$ de comprimento e $0,5 \mathrm{~cm}$ de diâmetro, localizadas na parede direita a $8,5 \mathrm{~cm}$ do assoalho e $10,5 \mathrm{~cm}$ distantes entre si (centro a centro). No centro desta parede, ao nível do piso, havia um bebedouro com capacidade de liberar $0,05 \mathrm{cc}$ de água. Sobre cada barra, uma lâmpada "olho-deboi" vermelha (12 watts) podia ser iluminada. Uma outra caixa, de dimensões semelhantes às anteriores, não continha barra ou qualquer outro manipulandum. Em todas, a parede frontal e o teto eram de acrílico transparente, sendo as demais paredes de aço inoxidável. O piso era composto por barras cilíndricas de aço inox, de 1/8" de diâmetro, espaçadas $1,3 \mathrm{~cm}$ entre si. A caixa sem manipulandum tinha o piso conectado a um estimulador de choque com alternador de polaridades (shock scrambler) de fabricação Lehind Valey 113-33.

Foi também utilizada uma caixa de alternação (shuttlebox) de acrílico, medindo 50,0 x 20,0 x 6,5 $\mathrm{cm}$, dividida em dois compartimentos iguais por uma parede central que continha um orifício medindo 6,0 $\mathrm{cm}$ de largura e 7,0 cm de altura (como uma "janela"), a $8,0 \mathrm{~cm}$ acima do assoalho. Cada compartimento tinha assoalho independente, preso no centro da caixa, podendo ser rebaixado na extremidade oposta: o peso do sujeito produzia a inclinação do piso, movimento que acionava um microswitch ${ }_{2}$ registrando automaticamente sua presença no compartimento. $\mathrm{O}$ piso, composto por barras cilíndricas de latão de $1 / 8$ " de diâmetro com espaçamento entre si de $1,3 \mathrm{~cm}$, era conectado a estimulador de choque com alternador de polaridades de fabricação BRS Foringer 901. Nessa caixa, a eletrificação do piso era independente em cada compartimento, de forma que o choque era liberado apenas no compartimento onde o sujeito se encontrava ao iniciar esse estímulo. Na superfície inferior do orifício na divisória central encontravam-se duas barras, semelhantes ao piso, conectadas ao estimulador de choques.

Todas as caixas experimentais eram isoladas entre si, acústica e visualmente, por câmaras construídas com compensado e fórmica. Um exaustor, localizado na parede lateral direita da câmara de isolamento, produzia a renovação do ar e um ruído constante que abafava ruídos externos.

A umidade relativa do ar foi registrada por um higrômetro localizado dentro da sala de coleta de dados e controlada por um desumidificador (marca Arsec, modelo 160M3-U) que a mantinha com um máximo de $70 \%$. A iluminação das caixas era proveniente do teto das câmaras de isolamento.

Os controles e registros foram realizados por computadores, utilizando softwares desenvolvidos especialmente para este experimento.

\section{Procedimento}

Os sujeitos foram divididos aleatoriamente em cinco grupos $(\mathrm{n}=8)$, submetidos a diferentes sessões de tratamento, espaçadas entre si por $24 \mathrm{~h}$. O procedimento constou de três fases: Fase 1 - reforço positivo, Fase 2 - choque incontrolável e Fase 3 - teste de fuga. Na Fase 1, animais de três grupos foram submetidos ao reforçamento positivo da resposta de pressão à barra, enquanto que os componentes dos dois grupos restantes foram mantidos no biotério sem qualquer tratamento experimental. Nessa fase, os animais passaram por uma sessão de modelagem da resposta de pressão à barra e 100 reforços em CRF, na caixa com uma barra. Em sessões sucessivas, foram colocados nas caixas com duas barras e submetidos a um dos seguintes esquemas de reforçamento: contínuo (grupo CRF), razão fixa (grupo FR) ou múltiplo-concorrente (grupo MULT). Os sujeitos dos grupos CRF e FR foram submetidos a cinco sessões de reforçamento, cujo encerramento se dava após 40 min ou 150 reforços, o que ocorresse primeiro. Para esses animais, as luzes sobre as barras permaneciam sempre apagadas, sendo consideradas para reforçamento as respostas de pressão a qualquer das barras. Para os animais do grupo CRF, toda resposta era seguida de reforço durante as cinco sessões. Para os sujeitos do grupo FR, na primeira sessão toda resposta produzia reforço (CRF), na segunda sessão o reforço seguia cada $4^{\mathrm{a}}$ resposta emitida (FR 4) e nas três sessões seguintes o reforço foi liberado a cada seis respostas (FR 6). Os animais do grupo MULT foram expostos a duas contingências concomitantes (FR e extinção), cada uma vigorando em uma das barras. O tempo de disponibilidade da contingência, denominado componente, era de 60 s. A cada componente, acendia-se a luz sobre uma das barras, ficando a outra apagada. Respostas na barra com luz acesa (SD) eram reforçadas em esquema FR, e na barra com luz apagada $(\mathrm{S} \Delta$ ) nenhuma resposta era seguida de reforço (extinção). Os componentes foram distribuídos de forma eqüitativa em ambas as barras ( 25 componentes de FR para cada barra), 
sendo sua alternância aleatória, com a única restrição de não haver mais do que três componentes iguais apresentados sucessivamente em uma mesma barra. Portanto, ao finalizar os $60 \mathrm{~s}$ de funcionamento da contingência em uma barra o mesmo esquema poderia ser mantido ou trocado pelo esquema alternativo. $\mathrm{Na}$ barra de reforçamento a intermitência foi introduzida gradualmente, de forma que nas duas primeiras sessões o esquema foi CRF/extinção e FR3/extinção, respectivamente, sendo mantido FR6/extinção nas sessões sucessivas. Para os três grupos, foi registrada a frequiência de respostas e total de reforços recebidos, sendo que no grupo MULT esse registro foi feito separadamente para cada barra (direita ou esquerda), indicando se a condição era de $\mathrm{SD}$ ou $\mathrm{S} \Delta$. O índice discriminativo (ID) foi calculado considerando-se respostas em SD / total de respostas. Assim, valores de ID em torno de 0,5 indicam que as respostas foram emitidas igualmente nas duas barras; valores próximos a 0,0 significam que as respostas dos sujeitos ocorreram em maior frequiência frente ao $\mathrm{S} \Delta$; valores próximos a 1,0 indicam que as respostas ocorreram com maior freqüência frente ao SD. Estipulou-se como critério mínimo de aprendizagem de discriminação o $\mathrm{ID}=0,8$. Para se atingir esse critério, foram realizadas 14 sessões.

$\mathrm{Na}$ Fase 2, os mesmos animais foram colocados na caixa sem qualquer manipulandum e submetidos a uma sessão de 60 choques elétricos de $1 \mathrm{~mA}, 10 \mathrm{~s}$ de duração fixa, liberados em intervalos médios de $1 \mathrm{~min}$ (com amplitude de variação entre $20 \mathrm{~s}$ e 100 s). Nessa sessão, respostas dos sujeitos não tiveram conseqüên- cias programadas, o que caracteriza esses choques como incontroláveis.

$\mathrm{Na}$ Fase 3, um teste de fuga foi realizado na caixa de alternação. Inicialmente os sujeitos permaneceram por 1 min sem qualquer tratamento, como período de adaptação. Em seguida, foram submetidos a 30 choques de $1 \mathrm{~mA}$, administrados em intervalos médios de 1 min (amplitude de variação de 20-100 s), com duração máxima de $10 \mathrm{~s}$, sendo a apresentação de cada choque chamada de tentativa. O choque era interrompido caso o sujeito saltasse de um compartimento para o outro, através do orifício central (resposta de fuga). Registrou-se a freqüência da resposta de fuga e o tempo de apresentação de cada choque, denominado latência de fuga. Caso a resposta de saltar não ocorresse até $10 \mathrm{~s}$, o choque era automaticamente desligado, a tentativa registrada como "falha" e a latência considerada como $10 \mathrm{~s}$.

Ao final das sessões de choque, as caixas eram limpas, retirando-se quaisquer detritos, utilizando-se, para isso, um pano umedecido com água e toalhas descartáveis umedecidas com álcool. Portanto, sempre que um novo sujeito era colocado na caixa, os odores liberados na situação de choque estavam reduzidos.

Dentre os animais que permaneceram no biotério durante a Fase 1, alguns foram expostos às Fases 2 e 3 (sessão de choques incontroláveis seguida pelo teste de fuga - grupo $\mathrm{CHI}$ ) e outros submetidos apenas à Fase 3 (teste de fuga - grupo ING).

O Quadro 1 resume o procedimento utilizado neste estudo.

Quadro 1. Resumo do procedimento empregado. Nas fases 1, 2 e 3, reforçamento positivo, choques incontroláveis e choques controláveis (fuga). Três grupos foram submetidos a todas as fases. Na Fase 1, foram expostos a esquemas de reforçamento contínuo (CRF), razão fixa (FR) ou múltiploconcorrente (MULT). Um grupo (CHI) passou apenas pelas Fases 2 e 3 e outro (ING) apenas pela Fase 3. Os sujeitos expostos à Fase 1 receberam um pré-treino de modelagem e 100 reforços em CRF. 0 intervalo entre sessões foi de $24 \mathrm{~h}$. IET = intervalo entre tentativas

\begin{tabular}{|c|c|c|c|}
\hline Grupos & $\begin{array}{c}\text { Fase } 1 \\
\text { Reforço positivo } \\
\text { (R - pressão à barra) }\end{array}$ & $\begin{array}{c}\text { Fase 2 } \\
\text { Choques incontroláveis }\end{array}$ & \begin{tabular}{|c|} 
Fase 3 \\
Choques controláveis (fuga) \\
(R - saltar)
\end{tabular} \\
\hline ING & & \multirow{5}{*}{$\begin{array}{c}60 \text { choques } \\
\text { (1 sessão) } \\
10 \mathrm{~s} \text { fixos } \\
1,0 \mathrm{~mA} \\
\text { IET médio } 60 \mathrm{~s} \\
(20-100 \mathrm{~s})\end{array}$} & \multirow{5}{*}{$\begin{array}{c}30 \text { choques } \\
\text { (1 sessão) } \\
10 \mathrm{~s} \text { max } \\
1,0 \mathrm{~mA} \\
\text { IET médio } 60 \mathrm{~s} \\
(20-100 \mathrm{~s})\end{array}$} \\
\hline $\mathrm{CHI}$ & & & \\
\hline CRF & $\begin{array}{c}\text { CRF } \\
\text { (5 sessões) }\end{array}$ & & \\
\hline FR & $\begin{array}{c}\text { CRF } \\
\text { (1 sessão) } \\
\text { FR } 4 \\
\text { (1 sessão) } \\
\text { FR } 6 \\
\text { (3 sessões) }\end{array}$ & & \\
\hline MULT & $\begin{array}{l}\text { CRF/extinção } \\
\text { (1 sessão) } \\
\text { FR } 3 / \text { extinção } \\
\text { (1 sessão) } \\
\text { FR 6/extinção } \\
\text { (12 sessões) }\end{array}$ & & \\
\hline
\end{tabular}


Os resultados do teste foram submetidos à Análise de Variância (ANOVA) two way para medidas repetidas e, quando necessário, as diferenças entre duplas foram analisadas pelo teste Tukey. Para alguns dados também foi utilizado o teste t de Student para amostras pareadas. O critério mínimo para que os resultados fossem considerados estatisticamente significantes foi $\mathrm{p}<0,05$.

\section{RESULTADOS}

$\mathrm{Na}$ fase de reforçamento positivo, todos os sujeitos emitiram sistematicamente a resposta de pressão à barra. Os animais reforçados em CRF mantiveram uma taxa relativamente constante ao longo das cinco sessões de reforçamento (em torno de 4,5 r/min), enquanto os animais reforçados em FR aumentaram suas taxas na medida em que a razão aumentou $(8,0$ e 10,0 R/min, no FR 4 e FR 6, respectivamente). Da mesma forma, os animais do grupo MULT apresentaram maiores taxas sob o esquema de razão mais elevada, sendo seus índices discriminativos crescentes ao longo das sessões: em média, apresentaram IDs de 0,46 e 0,93, na primeira e na última sessão, respectivamente, sendo que individualmente os animais apresentaram, na última sessão, ID mínimo de 0,85 e máximo 0,99.

$\mathrm{Na}$ sessão de teste, as latências da resposta de fuga foram analisadas em blocos de cinco tentativas (Figura 1). De uma maneira geral, esses resultados mostram que os animais expostos ao reforço positivo apresentaram latências de fuga menores que os sujeitos não expostos a esse tratamento, aproximando-se do padrão de desempenho dos animais ingênuos. No grupo ING, todos os sujeitos apresentaram latências relativamente baixas e decrescentes ao longo da sessão, sendo que apenas o Sujeito 3 apresentou aumento da latência no bloco final. Ao contrário, os animais do grupo $\mathrm{CHI}$ tiveram latências crescentes ao longo da sessão, sendo a única exceção o Sujeito 7 que apresentou no último bloco latência inferior aos blocos anteriores. Destacase que nesse grupo, metade dos sujeitos apresentou latências máximas (10 s) por diversos blocos sucessivos, mesmo depois de terem experimentado a contingência de fuga (latências abaixo de $10 \mathrm{~s}$ ). Nos demais grupos, que tiveram exposição ao controle do reforço positivo antes da sessão de choques incontroláveis, o desempenho foi variável: uma parte dos sujeitos aumentou e outra reduziu suas latências durante a ses- são, enquanto que alguns mantiveram latências pouco alteradas no decorrer dos blocos de tentativas.

A Figura 2 compara as médias dos grupos, considerando apenas as latências iniciais e finais. Os resultados apontam três conjuntos distintos de resultados: aumento, decréscimo acentuado e decréscimo suave. As poucas diferenças que aparecem no bloco inicial se acentuaram no final da sessão. Olhando-se apenas o bloco final, os padrões descritos geraram uma latência em torno de $2 \mathrm{~s}$, três delas em torno de $4 \mathrm{~s}$ e outra latência acima de $6 \mathrm{~s}$. Aparentemente, tais desempenhos relacionam-se com o tratamento prévio recebido: os animais não expostos a qualquer tratamento prévio (grupo ING) foram os que apresentaram as mais baixas latências, sendo os únicos que apresentaram acentuado declínio das mesmas durante a sessão, caindo de 4,5 s, no primeiro bloco, para menos da metade, ou seja, 2,2 s no bloco final. No sentido oposto, os animais do grupo submetido apenas aos choques incontroláveis antes do teste (grupo CHI), além de terem apresentado as maiores latências gerais foram também os únicos que apresentaram padrão de aumento dessas latências entre o primeiro e o último bloco: iniciaram a sessão com latência média de $6,0 \mathrm{~s}$ e terminaram com de 7,2 s. Por fim, os grupos submetidos ao reforçamento positivo na primeira fase tiveram latências gerais intermediárias, com pouco declínio entre começo e final de sessão: 4,8-4,0 s (grupo CRF), 4,9-4,7 s (grupo FR), 4,9-4,2 s (grupo MULT), no primeiro e no último bloco respectivamente.

Estatisticamente, foram significantes as diferenças obtidas em função do tratamento entre os grupos ING e CHI $(\mathrm{F}(1,14)=9,807, \mathrm{p}<0,05)$. As demais comparações estatísticas realizadas se mostram nãosignificantes.

A Figura 3 mostra que a distribuição de falhas ao longo dos blocos de tentativas acompanhou o padrão das latências. Os resultados individuais são mais homogêneos entre os sujeitos do grupo ING e do CHI: enquanto no primeiro grupo ocorreram poucas ou nenhuma falha, no segundo elas foram apresentadas por todos os sujeitos ao menos em três blocos de tentativas. Nos grupos submetidos ao tratamento inicial com reforçamento positivo, foram apresentados os três padrões de falha: nenhuma, poucas ou muitas falhas por repetidos blocos de tentativa. 

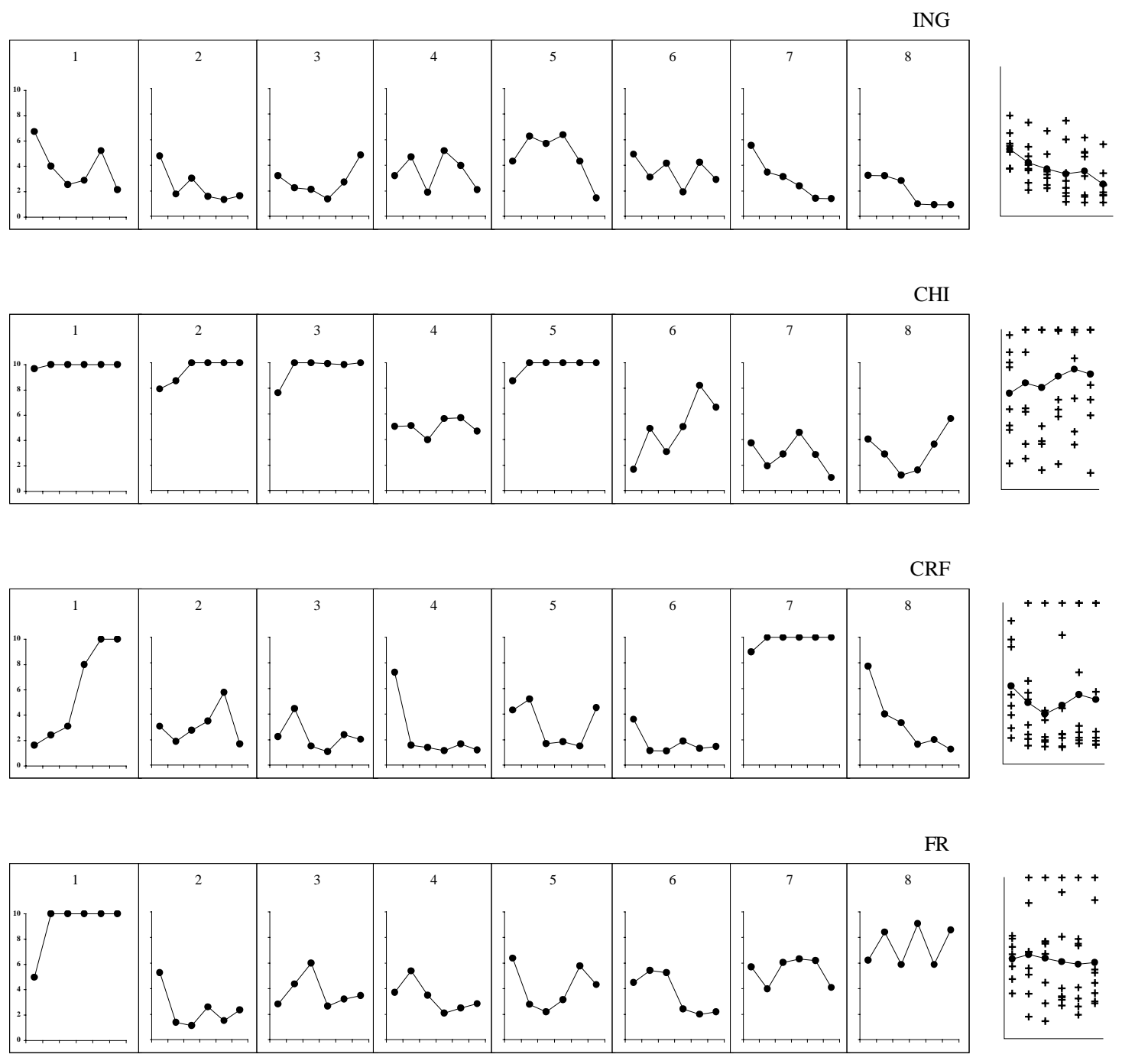

DISC

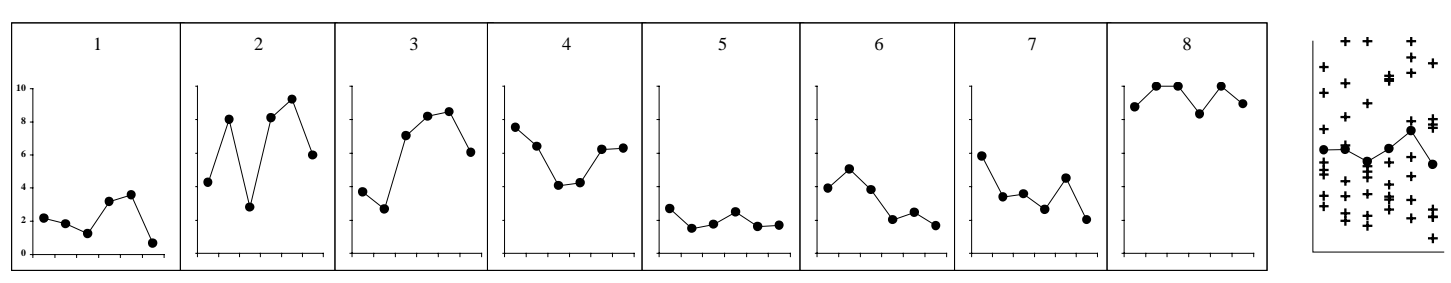

\section{Blocos de 5 tentativas}

Figura 1. Latências médias da resposta de fuga saltar apresentadas pelos oito sujeitos de cada grupo, plotadas em blocos de cinco tentativas. À direita, os pontos soltos ilustram os resultados individuais, enquanto a linha mostra a média do grupo. 


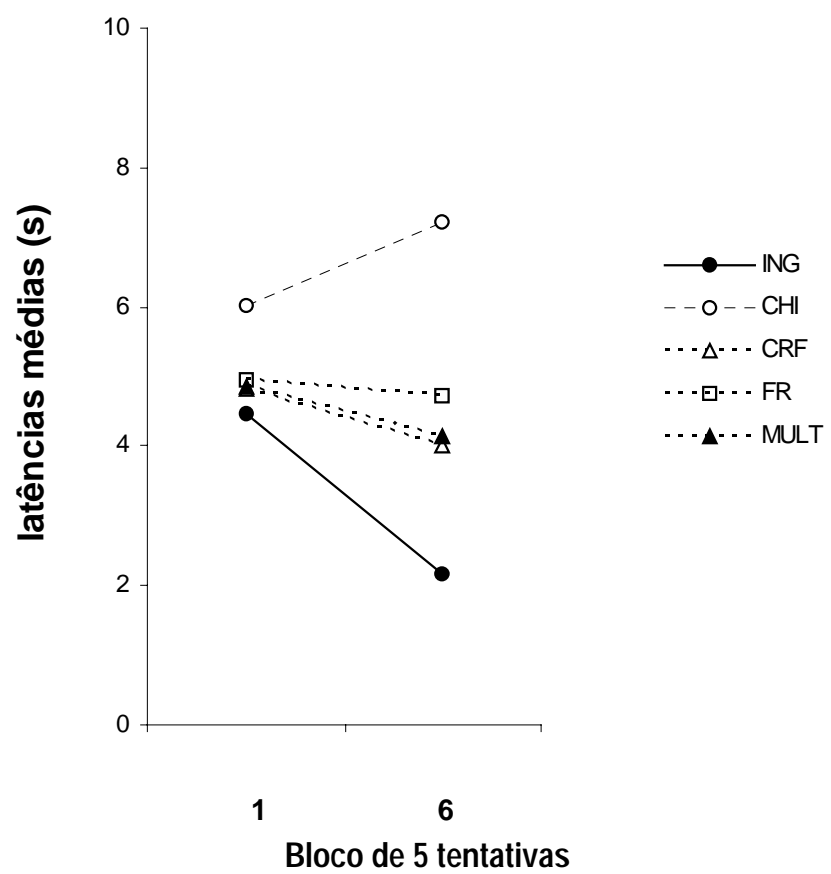

Figura 2. Latências médias da resposta de fuga saltar apresentadas pelos sujeitos dos diferentes grupos de tratamento no primeiro e no último bloco de cinco tentativas da sessão de teste.
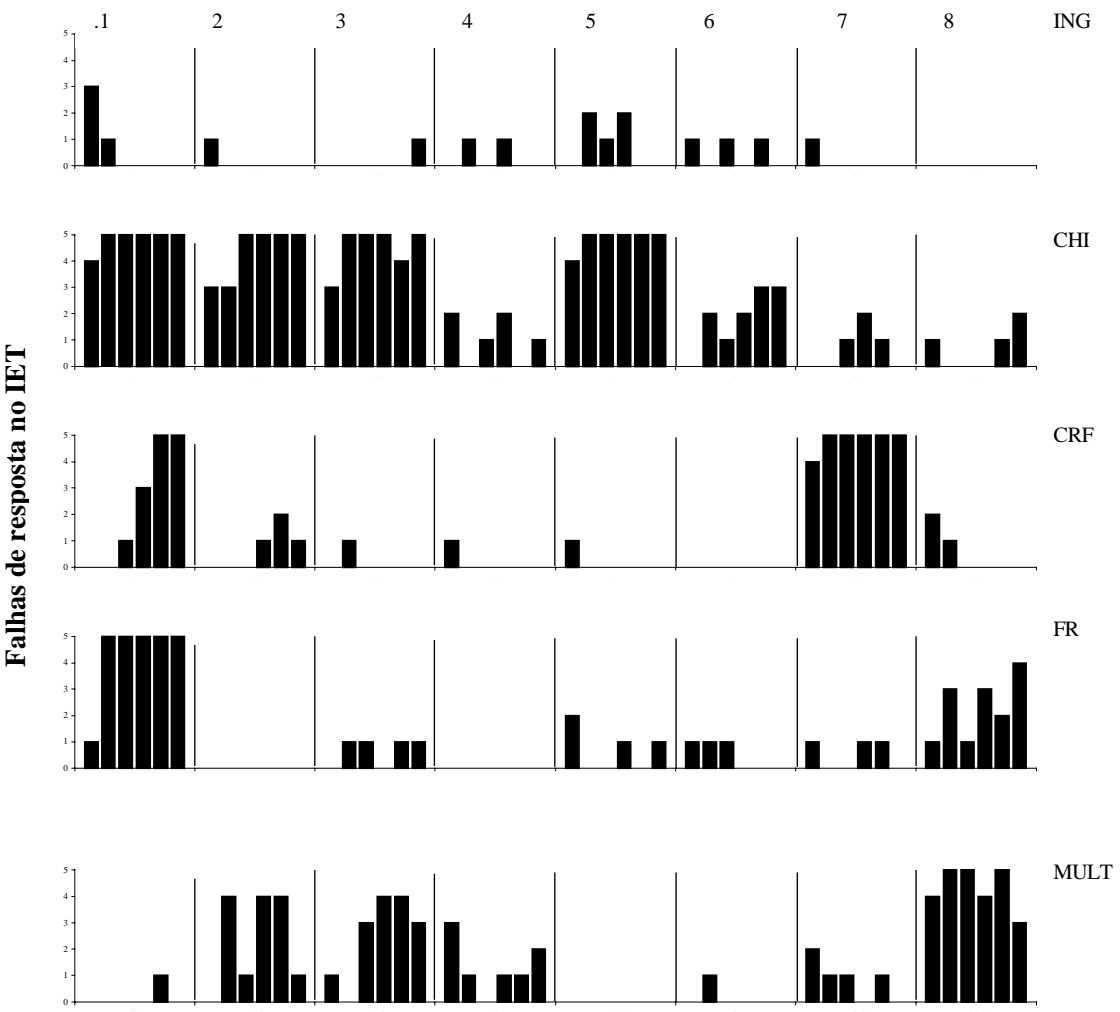

Bloco de 5 tentativas

Figura 3. Médias das falhas da resposta de saltar, em blowodeleiñdertentiatiksas, apresentadas pelos sujeitos de todos os grupos: ingênuo (ING), choque incontrolável, (CHI); reforçamento positivo em esquema contínuo (CRF), intermitente (FR) e múltiplo-concorrente (MULT). 


\section{DISCUSSÃO}

Os resultados da primeira fase mostraram que os sujeitos foram sensíveis às diferenças nas contingências de reforçamento empregadas: tanto a taxa de respostas como o comportamento discriminativo ficaram sob controle do esquema de reforçamento em vigor. Portanto, pode-se afirmar que, apesar de a experiência com controle sobre um aspecto do ambiente (a liberação do reforço) ter diferido entre os grupos, todos os animais submetidos à Fase 1 experimentaram a relação de dependência entre suas respostas de pressão á barra e a liberação da água.

Esse primeiro contato dos sujeitos com o reforço positivo reduziu os efeitos geralmente obtidos após exposição aos choques incontroláveis, permitindo uma aprendizagem de fuga estatisticamente igual à dos animais ingênuos. Esse resultado quantitativo corresponde ao efeito de imunização (Seligman \& Maier, 1967). Contudo, a análise qualitativa do desempenho desses sujeitos introduz algumas questões. Apesar dos animais previamente submetidos ao reforçamento positivo terem mostrado menor latência de fuga que os sujeitos expostos apenas aos choques incontroláveis, e nenhuma diferença estatística em relação aos animais ingênuos, os resultados mostram que eles praticamente não apresentaram declínio das suas latências ao longo da sessão. Como o declínio das latências é o dado que indica o estabelecimento do controle operante na contingência de fuga, não se pode afirmar que a resposta desses animais tenha ficado sob controle operante do reforçamento negativo. Assim, considerando-se que o desamparo é definido pela dificuldade de aprendizagem, se esses sujeitos não aprenderam fuga, seus resultados indicam desamparo. Portanto, Pode-se ter duas conclusões opostas a depender de quais critérios são aplicados aos presentes resultados.

Conceitualmente, o desamparo tem sido definido como um efeito de não aprendizagem ou de dificuldade de aprendizagem (Maier \& Seligman, 1976). Objetivamente, esses efeitos não são similares (ver Hunziker, 2005 ou Levis, 1976, para análise crítica dessa questão). A dificuldade de aprendizagem envolve emissão de respostas de fuga com latências decrescentes, porém significantemente superiores às apresentadas pelos sujeitos ingênuos. Por outro lado, a não aprendizagem de fuga envolve um dentre três padrões comportamentais: (1) não emissão de respostas, (2) emissão de respostas, porém cada vez mais demorada (latências crescentes ao longo da sessão), ou (3) emissão de respostas sem alteração das latências iniciais. Como todos esses padrões geram, ao final, latências superiores às apresentadas pelos sujeitos ingênuos, tem-se considerado que a significância estatística dessa diferença basta para caracterizar o desamparo. É com esse critério que os atuais resultados indicam imunização nos grupos previamente tratados com reforçamento positivo.

Apesar dessa adoção do critério quantitativo usualmente utilizado nos estudos sobre desamparo, não se pode deixar de apontar para a necessidade de mais análises sobre a adequação do mesmo. Será essa diferença quantitativa a mais adequada para a análise dos processos de aprendizagem envolvidos no desamparo e na imunização? A resposta a essa pergunta envolve uma análise longa que foge aos objetivos desse trabalho. Porém, nossos resultados permitem que se analise aqui a natureza das variáveis dependentes em estudo. Sob uma contingência de fuga, tanto a emissão da resposta como a sua latência são variáveis dependentes, sendo ambas inversamente relacionadas: maior número de respostas envolve, freqüentemente, menores latências, e vice-versa. Como a emissão da resposta é uma medida absoluta (tudo ou nada), e sua latência pode sofrer variações mais sutis (aqui, no contínuo entre 0,0 e 10,0 s), geralmente considera-se a latência como a variável mais sensível ao efeito de desamparo. Nesse estudo seguimos essa tendência, priorizando a análise das latências.

Contudo, contrariando essa tendência, Overmier (1985) sugeriu que a emissão de respostas de fuga e as suas latências são dois aspectos comportamentais distintos que podem sofrer diferentes efeitos do tratamento prévio recebido. Segundo ele, o tratamento do desamparo envolve duas variáveis independentes que são a incontrolabilidade e a imprevisibilidade dos estímulos, sendo esta última caracterizada pelo fato de que nenhum estímulo antecede sistematicamente a apresentação do choque. Nos estudos sobre o desamparo, a incontrolabilidade seria responsável pela baixa emissão da resposta de fuga, enquanto que a imprevisibilidade seria responsável pelo comportamento não ser modificado pela sua conseqüência, ou seja, mesmo após experimentar que a resposta produz o término do choque, o sujeito não apresentaria essa resposta com maior freqüência, nem reduziria suas latências frente a novos choques. Esses efeitos distintos foram denominados, respectivamente, de "déficit motivacional" e "déficit associativo" (Maier \& Seligman, 1976).

No atual experimento, os choques da fase de tratamento foram tanto incontroláveis quanto imprevisíveis. Assim, a análise de Overmier (1985) nos levaria 
a esperar que, no teste, seus efeitos fossem tanto de redução na freqüência da resposta de fuga como de aumento das suas latências. Isso de fato ocorreu nos animais do grupo CHI. Contudo, nos animais dos grupos pré-tratados com reforçamento positivo, a diferença mais visível foi sobre a emissão da resposta de fuga: houve redução das falhas, embora, na média, não tenha havido redução sistemática das latências ao longo da sessão. Esses resultados podem sugerir que o tratamento com reforçamento positivo imunizou os sujeitos contra o "déficit motivacional", mas não contra o "déficit associativo", ou seja, a imunização se deu para os efeitos da imprevisibilidade, mas não para os efeitos da incontrolabilidade. Embora os nossos resultados mostrem esse efeito bi-fatorial, não se pode dizer que eles confirmem a proposta de Overmier (1985), pois aqui não foi manipulada a previsibilidade dos choques. Essa é uma questão que fica para ser investigada futuramente em relação ao efeito de imunização.

Embora os resultados mostrem que estatisticamente todos os grupos pré-tratados com reforço positivo foram semelhantes ao grupo ING, o grupo que mais se aproximou dos resultados dos animais ingênuos foi aquele exposto ao reforçamento em CRF. Seguindo a análise de Overmier (1985), esse efeito talvez possa ser atribuído a diferenças quanto ao grau de controle e previsão estabelecidos na primeira fase (ver Seligman, Maier \& Solom, 1971, ou Catania, 1971, para análise dos conceitos de controle e previsão, relativos às relações $\mathrm{R}-\mathrm{S}$ e $\mathrm{S}-\mathrm{S}$, respectivamente). Por exemplo, na contingência CRF, a apresentação do reforço depende exclusivamente da emissão da resposta: a liberação do reforço $(\mathrm{S})$ segue $100 \%$ dos casos de emissão da resposta $(\mathrm{R})$, mas nunca ocorre na sua ausência (NR). Considerando-se que o controle é indicado pela diferença entre as probabilidades (p) da ocorrência de $\mathrm{S}$ em função de $\mathrm{R}$ ou de NR, pode-se dizer que o CRF é o esquema de reforçamento que maior grau de controle possibilita ao sujeito, pois a diferença entre $\mathrm{p}(\mathrm{S} / \mathrm{R})=1,0$ e $\mathrm{p}(\mathrm{S} / \mathrm{NR})=0,0$ é a diferença máxima possível entre duas probabilidades. Sendo essa relação sistemática, tal arranjo permite que a resposta do sujeito também adquira função de estímulo: a ocorrência da resposta (S1) sempre antecede a ocorrência do reforço (S2), ao mesmo tempo em que sua ausência (NS1) nunca antecede o reforço. Isso dá ao sujeito um alto grau de previsão sobre a liberação da água, pois a diferença entre $\mathrm{p}(\mathrm{S} 2 / \mathrm{S} 1)=1,0$ e $\mathrm{p}(\mathrm{S} 2 / \mathrm{S} 1)=0,0$ é a máxima possível. Diferentemente, os sujeitos do grupo FR tiveram uma relação R:S me- nor (6:1), que possibilita um grau de controle e previsão inferior ao do grupo anterior. No grupo MULT, o estímulo discriminativo só ocorria em metade da sessão, sinalizando uma relação de 6:1. Durante a outra metade da sessão, a sinalização indicava contingência nula (extinção), ou seja, ausência de relação R-S. Portanto, esses animais foram submetidos a graus de controle e previsão inferiores aos experimentados pelos animais do grupo CRF. Contudo, essa análise é puramente especulativa, uma vez que o delineamento experimental aqui utilizado não permite que se conclua sobre a relação direta entre graus de controle/previsão sobre o reforço positivo e o desempenho no teste de fuga. Novos experimentos devem ser executados para testar essa possibilidade.

Outro aspecto a ser analisado é o fato de que os animais dos grupos ING e CHI apresentaram desempenhos individuais uniformes, enquanto os sujeitos que tiveram "histórias conflituosas" - ou seja, experiência com controle sobre o reforço positivo na Fase 1, seguida da experiência com a incontrolabilidade dos choques na Fase 2 - tiveram maior variabilidade intragrupo, independentemente da contingência utilizada na fase inicial. Dentre esses últimos, houve padrões distintos: alguns indivíduos apresentaram redução das latências ao longo das tentativas (indicativo de aprendizagem de fuga, ou seja, imunização), outros mostraram latências estáveis ou aumento das mesmas durante a sessão, indicando insensibilidade ao reforçamento negativo (desamparo). Portanto, pode-se dizer que o tratamento prévio com reforçamento positivo foi efetivo para "imunizar" alguns sujeitos contra os efeitos dos choques incontroláveis, mas não para outros, o que gerou o resultado médio intermediário. $\mathrm{O}$ motivo dessa variabilidade intragrupo não fica claro. Sabe-se que, quando a variável independente manipulada é necessária e suficiente para produzir o fenômeno em estudo, ela abole as diferenças pré-experimentais entre os sujeitos, garantindo a uniformidade de resultados. Portanto, as diferenças intragrupo aqui obtidas sugerem que há outras variáveis que interferem no comportamento em estudo, além das variáveis independentes manipuladas. Seriam alguns sujeitos mais sensíveis à experiência de controle sobre o reforço positivo que à experiência de incontrolabilidade sobre os choques (e vice-versa), diferença essa que os levaria a apresentar padrões diversos mesmo tendo recebido igual tratamento? Se isso acontece, o que determinaria essa sensibilidade diferencial?

Uma possível interpretação pode ser relativa a diferenças genéticas. Há uma grande quantidade de 
estudos que demonstram que diversos comportamentos (operantes ou não) podem diferir em função da cepa dos ratos. Por exemplo, diferentes cepas apresentam diferenças nas respostas eliciadas por estímulos considerados estressantes (Belda, Márquez \& Armario, 2004; Faraday, 2002) e na a aprendizagem de fuga ou esquiva (Katzev \& Mills, 1974; Shurman \& Katzev, 1975). Especificamente nos estudos sobre desamparo, há indicações de que nem todos os sujeitos têm igual padrão de reação ao tratamento com a incontrolabilidade, sendo que, em alguns estudos, cerca de 33\% dos sujeitos não apresentaram o efeito (Seligman, 1975). Essa diferença levou à procura de determinantes genéticos, de forma que acasalamentos seletivos geraram cepas com probabilidade diferencial de desenvolvimento do desamparo aprendido (Anisman \& Waller, 1971; Shumake, Barrett\& Gonzalez-Lima, 2005). Nos estudos que utilizam choques elétricos, essas variações no padrão de aprendizagem são atribuídas às diferenças na reatividade aos choques, que seria geneticamente determinada (Anisman \& Waller, 1971; Katzev \& Mills, 1974). Segundo essa análise, uma aprendizagem de fuga ou esquiva pode se tornar mais provável se a resposta requerida para reforçamento for compatível com aquela que ocorre frente ao choque, e menos provável se ela for incompatível. Assim, ratos que tendem a apresentar "congelamento" (freezing) durante os choques teriam mais dificuldade de emitir a resposta de fuga ou esquiva que exigissem movimentação corporal do que os animais que se movimentam durante esses estímulos. Se aplicada ao atual estudo, essa análise nos permite sugerir que animais com diferentes graus de reatividade durante os choques talvez sejam afetados diferencialmente pelo treino inicial com reforço positivo, o que alteraria a probabilidade de apresentarem o efeito de imunização em função desse tratamento. $\mathrm{O}$ delineamento aqui utilizado não permite testar essa possibilidade, embora os seus resultados mostrem claramente a disparidade de efeitos entre sujeitos de um mesmo grupo de tratamento. Identificar as variáveis responsáveis por essa disparidade (genéticas ou outras) é uma tarefa para novos estudos.

Em resumo, os resultados do atual experimento sugerem que a exposição repetida ao reforçamento positivo pode reduzir os efeitos dos estímulos aversivos incontroláveis, evitando o desamparo aprendido. Essa demonstração tem desdobramentos importantes para a maior generalidade do efeito de imunização obtido no laboratório, assim como para as análises aplicadas que envolvem o desamparo como modelo animal de de- pressão (Seligman, 1975). Aparentemente, fica fortalecida a hipótese de que é a aprendizagem de controle, em si, e não a aprendizagem de controle de um único tipo de estímulo, que evita o desenvolvimento posterior do desamparo. Num primeiro passo de transição do laboratório para situações aplicadas, esses resultados podem sugerir pesquisas que avaliem o potencial "imunizador" da exposição repetida ao reforçamento positivo na prevenção contra a depressão.

Além disso, os resultados aqui apresentados também sugerem que a controlabilidade ou a incontrolabilidade dos estímulos podem não ser as únicas variáveis independentes críticas para o estudo do desamparo ou da sua imunização. A identificação de outras variáveis, de natureza contextual ou fisiológica, é um caminho a ser percorrido pelos pesquisadores da área.

\section{REFERÊNCIAS}

Alloy, L. B. \& Bersh, P. J. (1979). Partial control and learned helplessness in rats: control over shock intensity prevents interference with subsequent escape. Animal Learning and Behavior, 7, 157-164.

Anisman, H., Irwin, J., Beauchamp, C. \& Zacharko, R. (1983). Cross-stressor immunization against the behavioral deficits introduced by incontrolable shock. Behavioral Neuroscience, 97, 452-461.

Anisman, H. \& Waller, T. G. (1971). Effects of inescapable shock upon subsequent one-way avoidance learning in two strains of rats. Psychonomic Science, 24, 101-102.

Belda, X., Márquez, C., Armario, A. (2004). Long-term effects of a single exposure to stressing adult rats on behavior and hypothalamic-pituitary-adrenal responsiveness: comparison of two outbred rat strains. Behavioural Brain Research, 154, 399-408.

Brown, G. E., Howe, A. R. \& Jones, T. E. (1990). Immunization against learned helplessness in cockroach (Periplaneta Americana). Psychological Reports, 67, 635-640.

Catania, A. C. (1971). Elicitation, reinforcement and stimulus control. Em R. Glazer (Org.), The nature of reinforcement (pp. 196-220). New York: Academic Press.

Faraday, M.M. 2002). Rat sex and strain differences in responses to stress. Physiology and Behavior, 75, 507-522.

Hunziker, M. H. L. (2005). O desamparo aprendido revisitado: estudos com animais. Psicologia: Teoria e Pesquisa, 21, 131139.

Hunziker, M. H. L., Manfré, F. N. \& Yamada, M. T. (2006). Reforçamento positivo da variabilidade e da repetição imuniza contra o desamparo aprendido. Revista Brasileira de Análise do Comportamento, 2, 53-66.

Katzev, R. \& Mills, S. (1974). Strain differences in avoidance conditioning as a function of the classical CS-US contingency. Journal of Comparative and Psysiological Pschology, 87, 661671.

Kirk, R. C. \& Blampied, N. M. (1986). Transituational immunization against the interference effect (learned helplessness) by prior passive and active escape. The Psychological Record, 36, 203-214.

Interação em Psicologia, Curitiba, jul./dez. 2006, (10)2, p. 195-206 
Levis, D. J. (1976). Learned Helplessness: a reply and an alternative S-R interpretation. Journal of Experimental Psychology: General, 105, 47-65.

Maier, S. F. \& Seligman, M. E. P. (1976). Learned helplessness: theory and evidence. Journal of Experimental Psychology: General, 105, 3-46.

Mestre, M. B. A. \& Hunziker, M. H. L. (1996). O desamparo aprendido, em ratos adultos, como função de experiências aversivas incontroláveis na infância. Tuiuti: Ciência e Cultura, 6 (2), 25-47

Overmier, J. B. (1985). Toward a reanalysis of the casual structure of the learned helplessness syndrome. Em F. R. Brush \& J. B. Overmier (Orgs.), Affect, conditioning and cognition: Essays on the determinants of behavior ( $\mathrm{pp}$ 211-227). Hillsdale, NJ: Erlbaum.

Peterson, C., Maier, S. F. \& Seligman, M. E. P. (1993). Learned helplessness: a theory for the age of personal control. New York: Oxford University Press.

Ramirez, E., Maldonado, A. \& Matos, R. (1992). Attributions modulate immunization against learned helplessness in humans. Journal of Personality and Social Psychology, 62 (10), 139-146.

Seligman, M. E. P. (1975). Helplessness: on depression, development and death. San Francisco: Freeman.

Seligman, M. E. P. \& Maier, S. F. (1967). Failure to escape traumatic shock. Journal of Experimental Psychology, 74, 1-9.
Seligman, M. E. P., Maier, S. F. \& Solomon, R. L. (1971). Unpredictable and uncontrollable aversive events. Em E.F. Bush (Org.), Aversive Conditioning and Learning (pp. 347-400). New York: Academic Press.

Seligman, M. E. P., Rosellini, R. A. \& Kozak, M.J. (1975). Learned helplessness in the rat: time course, immunization, and reversibility. Journal of Comparative and Physiological Psychology, 88, 542-547.

Shumake, J., Barrett, D. \& Gonzalez-Lima, F. (2005). Behavioral characteristics of rats predisposed to learned helplessness: reduced reward sensitivity, increased novelty seeking, and persistent fear memories. Behavioural Brain Research, 164, 222 230.

Shurman, A. J. \& Katzev, R. D. (1975). Escape/avoidance responding in rats depends on strain and number of inescapable preshocks. Journal of Comparative and Physiological Psychology, 88, 548-553.

Yano, Y. \& Hunziker, M. H. L. (2000). Desamparo aprendido e imunização com diferentes respostas de fuga. Acta Comportamentalia, 8, 143-166.

\section{Nota:}

${ }^{1}$ Este trabalho recebeu apoio financeiro do CNPq (Bolsa Institucional de Mestrado e Bolsa Produtividade em Pesquisa), sendo parte da dissertação de Mestrado defendida pela segunda autora, na Universidade de São Paulo, em 2005. Ele foi parcialmente apresentado nas reuniões da ABPMC e ABA-Internacional, realizadas em Campinas/SP, em 2004. As autoras agradecem a leitura crítica de Cristiano Valério dos Santos.

\section{Sobre as autoras:}

Maria Helena Leite Hunziker: Professora Associada do Departamento de Psicologia Experimental da USP.

Raquel Scarpa Gebara Garcia de Lima: Bióloga, Mestre em Psicologia- área de concentração Psicologia Experimental.

Endereço para correspondência: Maria Helena Leite Hunziker - Universidade de São Paulo - Av. Prof. Mello Moraes, 1.721 05508-900 São Paulo - SP - Endereço eletrônico: hunziker@ lexxa.com.br. 